\title{
Autopercepción del estilo cognitivo profesional y dominancia cerebral en estudiantes de último año de secundaria*
}

\author{
Self-perception of professional cognitive style and brain \\ dominance in high school students
}

PP. 35-46

JULIÁN MANRIQUE A.**

REC: $17 / 08 / 2020$

Juan Felipe Martínez Flórez ***

ACEP: $21 / 11 / 2020$

\section{Resumen}

Este estudio busca examinar la relación entre aptitudes profesionales, perfil de estilo cognitivo (EC) y de dominancia cerebral (DC). Mediante un diseño correlacional se evaluó la autopercepción del estilo cognitivo profesional y la dominancia cerebral en 98 estudiantes de último año de secundaria. Se encontraron diferencias en el perfil técnicomanual, el cual lo seleccionaron mayormente los hombres, mientras que las mujeres optaron por los perfiles artístico-creativo y social-asistencial. Se observó un perfil de dominancia cerebral homogéneo entre los cuadrantes del hemisferio izquierdo $(\mathrm{HI})$ y del hemisferio derecho (HD), sin diferencias con respecto al género. Se obtuvieron correlaciones moderadas entre el perfil científico-investigadory el $\mathrm{HI}$, así como entre el perfil artístico-creativo y el HD. Las diferencias de género observadas en la elección de determinados perfiles profesionales no se sustentan en diferencias cognitivas. Las correlaciones observadas entre perfil profesional y dominancia cerebral guardan relevancia en términos de su correspondencia teórica y significancia estadística

* Artículo derivado de proyecto de investigación.

** Psicólogo Universidad del Valle, Magister Ciencia Cognitiva Universidad Ruhr Bochum, Docente Investigador Unicatólica Cali.

Email: jmanrique@unicatolica.edu.co, Orcid: https://orcid.org/0000-0003-2385-2372

*** Psicólogo Universidad del Valle, Magister Psicología Universidad del Valle, Doctor en Psicología Universidad del Valle.

Docente Investigador Unicatólica Cali. Email: jfmartinez@unicatolica.edu.co. Orcid: https://orcid.org/o000-0003-2914-0819 
Palabras clave: orientación vocacional, estilo cognitivo, dominancia cerebral.

\section{Abstract}

This study seeks to examine the relationship between professional skills, cognitive style profile (CS) and brain dominance (BD). Following a correlational design, the self-perception of professional cognitive style and brain dominance were evaluated in 98 last year high school students. Differences were found in the technicalmanual profile, which was chosen mostly by men, while women opted for the artistic-creative and social-assistance profiles. A homogeneous brain dominance profile was observed between the quadrants of the left hemisphere (LH) and the right hemisphere $(\mathrm{RH})$, without differences with respect to gender. Moderate correlations were obtained between the scientific-researcher profile and the $\mathrm{LH}$, as well as between the artisticcreative profile and the $\mathrm{RH}$. The gender differences observed in the choice of certain professional profiles are not based on cognitive differences. The correlations observed between professional profiles and brain dominance are relevant in terms of their theoretical correspondence and statistical significance.

Keywords: vocational orientation, cognitive style, brain dominance.

\section{Introducción}

La elección profesional es una decisión que tiene un alto impacto en la vida de un sujeto. En la cultura occidental y en ciertas clases sociales, la elección profesional se realiza a una edad temprana (16-20 años) que corresponde a los últimos años de escuela secundaria. Una decisión apresurada o poco satisfactoria tiende a ser una de las causas más prevalentes del bajo rendimiento académico y deserción en la educación universitaria (Zumárraga-Espinosa et al., 2018; Quinn, 2013; Spadies, 2019).
Como estrategia ante esta problemática se recurre a la orientación o consejería vocacional que busca determinar las preferencias y aptitudes personales en un marco objetivo a partirde la evaluación psicotécnica(Gottfredson, 2003). Sin embargo, este tipo de evaluación se suele enfocar en preferencias personales que pueden estar mediadas por la deseabilidad social, influencias contextuales o inmediatas. Se acusa la dificultad de poner en relación dichas preferencias con las capacidades y fortalezas de un sujeto a partir del estilo cognitivo (EC) y de dominancia cerebral (DC) que contribuya a una asertiva orientación vocacional.

En este trabajo se examinan las preferencias personales en la elección profesional a partir del EC y los perfiles de DC de un grupo de estudiantes de último año de secundaria de un colegio privado de bachillerato industrial de la ciudad de Cali, Colombia con el fin de establecer qué tan relacionadas están las preferencias personales con sus perfiles cognitivos de aptitud profesional y dominancia cerebral.

\section{Estilo cognitivo}

El constructo de EC propone que hay diferencias individuales en la manera en que los sujetos procesan información (Genovese, 2005; Kozhevnikov, 2007). El EC se define como esquemas o estrategias habituales que determinan los modos de operacionalizar el procesamiento yla solución de problemas (Ramiro et al., 2010). El constructo de EC se originó en los años 60 como una forma integrar resultados investigativos y teoría de la cognición en un marco que permitiese una aplicación práctica en contextos como la educación o las organizaciones (Tinajero Páramo, 2013). Este constructo cuenta con diferentes propuestas conceptuales, modelos teóricos y pruebas experimentales, con lo cual propicia gran pluralidad de términos, resultados e interpretaciones (Coffield et al. 2004; Cools, 2009). 
Una apuesta por la integración son los datos provenientes de la neurociencia. Evidencia experimental (Bendall et al., 2016; Höffler et al., 2017; Kraemer et al., 2009; Shin y Kim, 2015) sugiere que el constructo de EC puede estar soportado en la especialización hemisférica y funcional del cerebro, con lo que propone que el ejercicio de la cognición conlleva desarrollar la preferencia por un EC determinado.

El modelo Hermann (1991; 1996; 2009) propone que el EC se puede representar en cuatro cuadrantes: A) racional-lógico, B) práctico-organizador, C) relacionalcomunicador e D) intuitivo-visionario y se encuentran soportados en la especialización funcional del cerebro (ver figura 1). Así pues, se esperaría en las personas dedicadas a las ciencias naturales un ajuste del perfil que favorece a los cuadrantes A y B, mientras que el cuadrante $D$ se vería favorecido en los artistas. De igual forma, se supone que el cuadrante C sería dominante en los profesionales de ciencias sociales y el $B$ en los administradores (Gardié, 2000).

Figura 1.

Modelo de cerebro total (Hermann,1991)

\begin{tabular}{|c|c|}
\hline Cuadrante A & Cuadrante D \\
Lógico & Intuitivo \\
Crítico & Simultáneo \\
Realista & Integrador \\
Analítico & Espacial \\
Cuantitativa & Imaginativo \\
\hline Cuadrante B & Cuadrante C \\
Administrador & Comunicativo \\
Secuencial & Expresivo \\
Detallista & Musical \\
Planificador & Espiritual \\
Conservador & Empático \\
\hline
\end{tabular}

Fuente: Adaptado por los autores de Hermann (1991)

\section{Dominancia cerebral}

Al Herrmann (2009) medir la actividad eléctrica cerebral con electroencefalografía (EEG) reportó que en tareas de dibujo el hemisferio derecho (HD) muestra mayor actividad eléctrica (actividad beta), mientras que el hemisferio izquierdo (HI) se registraba inactivo (actividad alfa). En contraposición, al realizar tareas de aritmética se registró que el HI estaba en un estado beta alto mientras que el HD estaba en un estado beta inferior.

Resultados con resonancia magnética funcional (RMF) reportan sustratos neurales específicos para el razonamiento deductivo y matemático (Kroger et. al. 2008) al relacionar un perfil de razonamiento deductivo con actividad en la corteza prefrontal derecha y el lóbulo parietal inferior, mientras un perfil de tipo matemático se relaciona con la corteza prefrontal izquierda y el lóbulo parietal superior. En esta misma línea, EttingerVeenstra et al. (2010) señala actividad dominante en el HD en sujetos con mejor rendimiento en tareas de interpretación lingüística. Por otro lado, sujetos con un EC visual registran mayor actividad en el giro fusiforme derecho, mientras que sujetos con un $\mathrm{EC}$ verbal en el giro supramarginal del $\mathrm{HI}$ (Kraemer et al., 2009). Por último, sujetos con un estilo de control-ejecutivo muestran mayor actividad en la corteza prefrontal y el precuneo del HI (Shin y Kim, 2015).

En relación con lo anterior, se intenta establecer si las diferencias en el funcionamiento cerebral se relacionan con determinados perfiles de estilo cognitivo (Blazhenkova y Kozhevnikov 2009; Yarlequé et. al. 2018; Velásquez et al., 2013). Incluso, autores como Gardié (2000) proponen que la DC y los EC se corresponden con las características que definen los atributos de 
una profesión. En este trabajo se aborda este tema desde la perspectiva previa. Es decir, el objetivo es determinar si las preferencias personales y el estilo cognitivo están en relación con un perfil de dominancia cerebral al momento de realizar la elección de carrera.

\section{Método}

El estilo metodológico de la investigación se describe bajo la sección de procedimiento a continuación.

\section{Procedimiento}

Mediante un diseño transversal de tipo descriptivo-correlacional se buscó establecer la relación entre el perfil de estilo cognitivo (EC), perfil de aptitud profesional y perfil de dominancia cerebral (DC) de los participantes del estudio. Los instrumentos usados para medir cada variable los aplicaron estudiantes de último año de Psicología entrenados en la administración de pruebas, junto a dos psicólogos docentes investigadores. La aplicación se realizó en tres sesiones/días diferentes y se separó a los sujetos en tres grupos según su pertenencia a cursos (11-1, 11-2, 11-3).

Durante la evaluación, los estudiantes de la muestra se asignaron al azar en dos grupos según la lista de asistencia, de manera que la aplicación se realizó paralelamente en dos salones con no más de 15 sujetos, acompañados por dos estudiantes de Psicología y un psicólogo docente. En cada sesión los participantes diligenciaron primero cuestionario Explora que más adelante se detalla durante 30 minutos y seguidamente el TDTCT durante 15 minutos. Se usaron hojas de respuesta en papel y lápiz. Para el cuestionario Explora se realizó la calificación automática mediante la interfaz de TEA ediciones y con el TDTCT se realizó de manera manual. Para el procesamiento de la información se uso el software estadístico SPSS versión 23.o. Se utilizaron tablas de distribución de frecuencias, categorización de respuestas en tablas de contingencia y se emplearon análisis estadísticos de prueba de hipótesis T-student, Anova de un factor, se asumió la igualdad de varianza y un nivel de significancia $P=0,05$.

\section{Participantes}

Participaron 98 sujetos de grado $11^{\circ}$ de educación secundaria de un colegio arquidiocesano privado de la ciudad de Cali, Colombia, entre ellos $51 \%(\mathrm{~N}=50)$ de género femenino y $49 \%(\mathrm{~N}=48)$ masculino, con una edad media de 16 años y $D E=7,0$. El centro educativo es de carácter privado, pero con un enfoque social incluyente, es decir que es un colegio que atiende población de estratos 1 a 3. Los núcleos familiares de los participantes de este estudio son en su mayoría de estos estratos $(1=7,7 \%, 2=50$ $\%$ y $3=30,8 \%$ ) y presenta un número inferior de participantes de estratos 4 (3,8\%) y 5 ( 7 $\%)$. El colegio que sirvió de marco muestral tiene una orientación de bachillerato industrial y cuenta con la instalación de talleres de mecánica industrial, tecnología e informática, y artes plásticas. Los padres y/o responsables de los participantes menores de edad fueron informados de los objetivos del estudio y aceptaron firmar voluntariamente el consentimiento para permitir la participación de sus hijos, quienes así mismo firmaron el asentimiento informado. Para el análisis y manejo de la información se siguieron los protocolos del acuerdo de Helsinki (2015) y la Ley 1090 de 2006 del colegio colombiano de psicólogos (Congreso de la Republica, 2006). 


\section{Instrumentos}

Cuestionario para la orientación vocacional y profesional, Explora

El test Explora (Martínez-Vicente y Santamaría, 2013; Martínez-Vicente, 2016) se diseñó a partir de los tipos y modelos ambientales propuestos por Holland (1992; 1997). Contiene 180 ítems que evalúan seis campos profesionales: a) técnicomanual (TM); b) científico-investigador (CI); c) artístico-creativo (AC); d) socialasistencial (SA); e) empresarial-persuasivo (EP); y f) oficina-administración (OA). Están distribuidos en tres secciones: 1) actividades y profesiones con 18 ítems (9 de actividades y 9 de profesiones) por campo profesional que permiten al sujeto expresar su preferencia por actividades y profesiones, e indicar la preferencia, disgusto o indecisión por realizar la actividad o profesión propuesta; 2) aptitudes y destrezas, contiene 9 ítems por campo profesional que indican el dominio de habilidades y destrezas, señalan así si considera que la tiene o que podría aprenderla si no la posee o se ve incapaz de aprenderla o indecisión; 3) características de personalidad, que contiene 3 ítems por campo profesional. En esta sección el sujeto debe considerar una serie de adjetivos y valorar si describen su forma de ser, si no considera que sea así o si no se puede decidir.

La aplicación del Explora permite obtener información adicional que permite determinar la probabilidad de realizar elecciones profesionales adecuadas y estables que garanticen la futura satisfacción y rendimiento laboral. Entre dichos índices complementarios se encuentra el estilo de respuesta que se refiere a las dudas (indecisión como opción de respuesta en cada pregunta) e inconsistencias, los se obtiene a partir de contrastar las puntuaciones (altas o bajas) en diferentes perfiles profesionales obtenidos. Así pues, se infiere que está tan confundido quien no tiene ninguna idea de qué carrera estudiar como aquel que tiene muchas opciones con igual preferencia.

Si bien el Explora se puede usar con diferentes tipos de poblaciones, se enfatiza su uso y efectividad con la población de educación secundaria (Martínez-Vicente, 2019), puesto que es en esta etapa en la cual se busca la consolidación de los intereses vocacionales.

\section{Cuestionario de dominancia cerebral}

El perfil personal de estilo de pensamiento - Test de Diagnóstico Teoría Cerebro Total (TDTCT) Jiménez, 2000 corresponde a una adaptación a Colombia de la prueba Cerebro Total desarrollada por Hermann (1991). Este instrumento valora el estilo personal de procesamiento de información en el cerebro, lo cual da lugar a perfiles de dominancia cerebral en cuatro cuadrantes: 1) dominante superior izquierdo (DSI); dominante inferior izquierdo (DII); dominante superior derecho (DSD); y dominante inferior derecho (DID), así como otras combinaciones de dominancia doble o triple. Es un cuestionario de autoevaluación en relación con actividades de estudio y de la vida diaria (50 en total, 10 por cada cuadrante cerebral), resuelto en escala tipo Likert de 5 (lo que hago mejor) a 1 (lo que hago peor).

\section{Resultados}

\section{Perfiles cognitivo profesionales}

Los resultados generales de los perfiles del test Explora muestran que de la muestra total $16,22 \%(n=16)$ señala un EC coincidente con un perfil para AC, el $16,87 \% \quad(n=16)$ para $\mathrm{Cl}$, el 24,16\% $(n=23)$ para EP; el $11,8 \%$ $(n=12)$ con OA; el $19,87 \%(n=19)$ con SA y el $11,02 \%(n=11)$ para TM. Por otro lado, la 
media del resultado por género (ver tabla 1), no muestra diferencias significativas en los perfiles del Explora, salvo en el perfil TM en el que el grupo femenino (GF) obtuvo un puntaje medio de 1,63 vs grupo masculino (GM) de 2,50 ( $\mathrm{t}=2,32 ; p=0,02)$. En el perfil de $A C$ la media del $G F$ fue de $3,40(D E=2,5)$ vs $\mathrm{GM}=2,61(\mathrm{DE}=2,0)(\mathrm{t}=-1,58 ; p=0,11)$.

Incluso cuando se observa una diferencia cercana a la significancia estadística en este perfil, se podría afirmar que las capacidades cognitivas y aptitudes personales de los estudiantes que tienden a escoger carreras afines a dicho perfil (AC) están presentes tanto en hombres como en mujeres. Contrariamente, el perfil TM adscribe aptitudes y capacidades que son, generalmente, más proclives a identificarse con el género masculino.

Como se mencionó anteriormente, en los demás perfiles que evalúa el Explora no se registran diferencias significativas por género. Para el perfil de $\mathrm{Cl}$ la media del $\mathrm{GF}=2,80(\mathrm{DE}=2,2)$ vs $\mathrm{GM}=3,50(\mathrm{DE}=2,3)(\mathrm{t}=$ 1,3; $p=0,16)$; para el perfil de SA la media del $\mathrm{GF}=3,90(\mathrm{DE}=2,2)$ vs $\mathrm{GM}=3,48(\mathrm{DE}=2.2)(\mathrm{t}=$ $-0,84 ; p=0,39)$; en el perfil de EP la media del $\mathrm{GF}=4,58(\mathrm{DE}=2,4)$ vs $\mathrm{GM}=4,40(\mathrm{DE}=2,4)(\mathrm{t}=$ $-0,34 ; p=0,73) ; y$ en el perfil de OA la media del $\mathrm{GF}=2,48(\mathrm{DE}=2,1)$ vs $\mathrm{GM}=1,91(\mathrm{DE}=1,7)$ $(\mathrm{t}=-1,31 ; p=0,19)$.

\section{Tabla 1.}

Resultados del test Explora

Perfiles test Explora

\begin{tabular}{ll}
\hline Técnico manual & 2,50 \\
\hline Artístico creativo & 2,61 \\
\hline Científico investigador & 3 \\
\hline Social asistencial & 3 \\
\hline Empresarial persuasivo & 4 \\
\hline Oficina administración & 1, \\
\hline Fuente: elaboración propia \\
Dominancia cerebral
\end{tabular}

Los resultados en el TDTCT muestran que el $24,51 \%(n=24)$ de los participantes muestra un perfil coincidente para DSI y el 25,59 $\%(n=25)$ cuadrante DII. Para la dominancia en el hemisferio derecho un $24,84 \%(n=24)$ muestra dominancia preferente para DSD y un $25,06 \%(n=25)$ para DID. En el TDTCT no se registran diferencias significativas en la comparación de los puntajes medios entre el GF y GM (ver tabla 2). En el cuadrante DSI el puntaje medio del $\mathrm{GF}=32,9$ vs $\mathrm{GM}=34,8(\mathrm{t}=$ $1,24 ; p=0,82)$; en el cuadrante DII la media de $\mathrm{GF}=35,6$ vs $\mathrm{GM}=35.2(\mathrm{t}=-0,32 ; p=0,60)$; para el cuadrante DID la media del $\mathrm{GF}=34,6$ vs $\mathrm{GM}=35,1(\mathrm{t}=0,40 ; p=0,93)$; finamente, en el cuadrante DSD la media del $\mathrm{GF}=33,9$ vs $\mathrm{GM}=34,4(\mathrm{t}=0,38 ; p=0,72)$. Al respecto, es menester apuntar que, si bien existen datos de contraste entre la estructura y función cerebral de hombres y mujeres, los resultados son más inconsistentes cuando se infieren correlatos de estas diferencias en la función cognitiva. Justamente, se podría argumentar que siendo el TDTCT un cuestionario actitudinal, no resulta lo suficientemente sensible para registrar una diferencia entre géneros con respecto a la especialización cognitiva que se atribuye a cada cuadrante y hemisferio cerebral. 
Tabla 2.

Resultados del test de dominancia cerebral

\begin{tabular}{lllll}
\hline Cuadrantes cerebrales & $\begin{array}{l}\text { Grupo masculino (GM) } \\
\text { M (DE) }\end{array}$ & $\begin{array}{l}\text { Grupo femenino (GF) } \\
\text { M (DE) }\end{array}$ & t-test & Valores p \\
\hline Superior izquierdo & $34,8(6,8)$ & $32,9(7,1)$ & 1,24 & 0,82 \\
\hline Inferior izquierdo & $35,2(2,0)$ & $35,6(2,5)$ & $-0,32$ & 0,60 \\
\hline Inferior derecho & $35,1(6,4)$ & $34,6(5,8)$ & 0,40 & 0,93 \\
\hline Superior derecho & $34,4(6,4)$ & $33,9(6,3)$ & 0,38 & 0,72 \\
\hline
\end{tabular}

Fuente: elaboración propia

\section{Correlación perfil profesional/dominancia cerebral}

La correlación entre los EC resultantes del test Explora y la dominancia por cuadrantes en el TDTCT muestra correlaciones positivas de intensidad moderada y de significancia estadística (ver tabla 3).

\section{Tabla 3.}

Correlación perfil profesional con dominancia cerebral

\begin{tabular}{|c|c|c|c|c|c|c|c|c|c|}
\hline & $\mathrm{TM}$ & $\mathrm{Cl}$ & $A C$ & SA & EP & $\begin{array}{l}\text { Sup. } \\
\text { Izq. }\end{array}$ & Inf. Izq. & Inf. Der. & Sup. Der. \\
\hline \multirow{2}{*}{ TM } & r de Pearson & - & & & & & & & \\
\hline & Valor $\mathrm{p}$ & - & & & & & & & \\
\hline \multirow{2}{*}{$\mathrm{Cl}$} & r de Pearson & 0,366 & - & & & & & & \\
\hline & Valor $p$ & 0,001 & - & & & & & & \\
\hline \multirow{2}{*}{$A C$} & r de Pearson & 0,201 & $-0,016$ & - & & & & & \\
\hline & Valor $\mathrm{p}$ & 0,047 & 0,879 & - & & & & & \\
\hline \multirow{2}{*}{ SA } & r de Pearson & 0,021 & 0,262 & 0,227 & - & & & & \\
\hline & Valor $\mathrm{p}$ & 0,838 & 0,009 & 0,025 & - & & & & \\
\hline \multirow{2}{*}{ EP } & r de Pearson & 0,173 & $-0,030$ & $-0,045$ & 0,277 & & & & \\
\hline & Valor $p$ & 0,088 & 0,773 & 0,663 & 0,006 & & & & \\
\hline \multirow{2}{*}{$\mathrm{OA}$} & r de Pearson & 0,123 & $-0,062$ & $-0,052$ & 0,280 & - & & & \\
\hline & Valor $\mathrm{p}$ & 0,227 & 0,543 & 0,612 & 0,005 & - & & & \\
\hline \multirow{2}{*}{$\begin{array}{l}\text { Sup. } \\
\text { Izq. }\end{array}$} & r de Pearson & 0,240 & 0,419 & 0,038 & 0,254 & 0,165 & - & & \\
\hline & Valor $\mathrm{p}$ & 0.017 & 0,001 & 0,708 & 0,011 & 0,105 & - & & \\
\hline \multirow{2}{*}{$\begin{array}{l}\text { Inf. } \\
\text { Izq. }\end{array}$} & r de Pearson & 0,110 & 0,107 & 0,188 & 0,133 & 0,229 & 0,565 & - & \\
\hline & Valor $\mathrm{p}$ & 0,281 & 0,293 & 0,064 & 0,193 & 0,023 & 0,001 & - & \\
\hline \multirow{2}{*}{$\begin{array}{l}\text { Inf. } \\
\text { Der. }\end{array}$} & rde Pearson & 0,077 & 0,016 & 0,172 & 0,443 & 0,230 & 0,343 & 0,527 & - \\
\hline & Valor $\mathrm{p}$ & 0,452 & 0,879 & 0,090 & 0,001 & 0,023 & 0,001 & 0,001 & - \\
\hline \multirow{2}{*}{$\begin{array}{l}\text { Sup. } \\
\text { Der }\end{array}$} & r de Pearson & 0,221 & 0,095 & 0,445 & 0,081 & $-0,037$ & 0,504 & 0,526 & 0,428 \\
\hline & Valor $\mathrm{p}$ & 0,029 & 0,351 & 0,001 & 0,426 & 0,716 & \%... 0,001 & 0,001 & 0,001 \\
\hline
\end{tabular}

Fuente: elaboración propia

En primera instancia, se observa una correlación significativa entre el estilo $\mathrm{Cl}$ y el DSI $r=0,41(p-0,00)$. En esta misma línea, se registra correlación positiva entre AC y DSD $r=$ $0,44$ ( $p$ : 0,00$)$; entre SA y DID $r=0,44(p \div 0,00)$ y entre EP y DII $r=0,29(p ; 0,00)$. En segunda instancia, se registran correlaciones positivas con una baja intensidad, pero con significancia estadística. Se observa una doble correlación entre el estilo TM con DSI $(r=0,24 ; p ; 0,01)$ y $\operatorname{DSD}(r=0,22 ; p ; 0,02)$; de igual forma entre $\mathrm{OA}$ y DII $(r=0,22 ; p \div 0,02)$ y DID $(r=0,23 ; p \div 0,03)$. 
Por último, entre la variable de edad y los dominios de dudas e inconsistencias en el test Explora (ver tabla 4) se registra la tendencia de que, a menor edad, mayor es el valor de los tópicos de dudas $(r=-0,19$; $\mathrm{p}$ contraria con el factor de inconsistencias $(r=0,20$ p $: 0,04)$, el cual alcanza una significancia estadística. Lo anterior quiere decir que los adolescentes de mayor edad conciben diferentes opciones de orientación vocacional, pero quizá no tienen aún claridad respecto cuál elección se ajusta mejor a sus aptitudes y capacidades cognitivas.

\section{Tabla 4.}

Correlación entre la edad y las dudas e inconsistencias en el test Explora

\begin{tabular}{lllll}
\hline Variable & & Edad & Inconsistencias & Dudas \\
\hline Edad & $\begin{array}{l}\text { R de Pearson } \\
\text { Valor } p\end{array}$ & 1 & 0,204 & $-0,19$ \\
& R de Pearson & 0,204 & 1 & 0,06 \\
\hline Inconsistencias & Valor $p$ & 0,04 & & 0,98 \\
& R de Pearson & $-0,19$ & 0,98 & 0,33 \\
\hline Dudas & Valor $p$ & 0,06 & 0,33 & 1 \\
& & & & \\
\hline
\end{tabular}

Fuente: elaboración propia

\section{Discusión}

En este trabajo se buscó describir y 42 determinar la correlación entre los EC profesionales y los perfiles de dominancia cerebral en una muestra de estudiantes de último año de secundaria. Se observaron diferencias con significancia estadística en el EC profesional TM entre el GF y GM. Diversos autores (Figueroa y Ortega 2010; Navarro y Casero, 2012; Rodríguez, Peña y Pérez, 2016) concuerdan en que las diferencias de género construyeron y delimitaron espacios sociales entre lo masculino y lo femenino en la sociedad, lo que conllevó que persistan distinciones por género en la elección profesional. El género masculino tiende a elegir e identificarse con carreras asociadas al rol manual, técnico o investigador mientras el género femenino a aquellas relacionadas con el cuidado, lo artístico o lo expresivo.

En cuanto a los perfiles de dominancia cerebral en el TDCT, no se registraron diferencias significativas en los puntajes. Lo anterior podría soportar que las diferencias observadas en los EC profesionales se deben a influencias de índoles histórico-social y no de orden biológico-estructural. En este sentido, es importante reseñar al autor Lesley Rogers (2001) quien en su libro Sexing the brain argumenta que las experiencias vividas (tales como deportes preferidos, materias escolares recibidas, influencia parental) proporcionan influencias en la socialización de ambos sexos, lo cual finalmente se podría relacionar con la preferencia por distintas orientaciones vocacionales, acordes con la educación social y cultural recibida según el género.

Ahora bien, las correlaciones observadas entre el EC y la DC, si bien no registran una fuerza alta, guardan relevancia en términos de su correspondencia teórica, alta significancia estadística y que no se encuentran reportadas en la literatura. La correlación positiva entre $\mathrm{Cl}$ y DSI se corresponde con lo reportado previamente en estudios de dominancia cerebral (Kraemer et al., 2009; Shin y Kim, 2015) que asignaron al hemisferio izquierdo, específicamente a la corteza frontal izquierda, funciones relacionadas con el procesamiento secuencial, análisis, planificación, abstracción. En esta misma línea, es coincidente la 
correlación entre AC y DSD. Evidencia reciente (Aberg et al., 2016; Goldberg, 2019; Shi et al., 2017) sugiere que el involucramiento del hemisferio derecho -particularmente la corteza frontal derecha- en el proceso creativo no es únicamente funcional, sino que parece ser además bioquímico, en función de la baja concentración de dopamina en este hemisferio y la prevalencia del circuito noradrenérgico correlacionado con procesos como el pensamiento divergente e innovación (Khalil et al., 2019).

Se registraron además correlaciones en áreas posteriores de SA con DID, y EP con DII. En ambos casos estamos hablando de EC profesionales que se relacionan con procesos de orden afectivo/social como la empatía, reconocimiento de emociones y teoría de la mente. Si bien la organización de estos procesos es de tipo "circuito", en el caso de la empatía involucra el giro supramarginal, la juntura parietal temporal dorsal izquierda y corteza cingulada posterior izquierda, mientras que en el caso de la teoría de la mente se relaciona con la juntura parietal temporal derecha y corteza cingulada derecha (Kanske 2018; Stietz et al., 2019; Schurz et al., 2014).

Las correlaciones dobles observadas entre TM y DSI/ DSD tienen sustento en la evidencia de que la función motora se encuentra organizada en el lóbulo frontal de ambos hemisferios (Fountoulakis et al., 2019). Por otro lado, la correlación doble de OA con DII y DID permite pensar que un EC profesional organizacional administrativo puede depender de la interrelación de diferentes habilidades cognitivas organizadas en estos dos cuadrantes. En este sentido, la teoría del cerebro total de Hermann $(1991,2001)$ enfatiza en que, a partir de la interacción de los cuadrantes se puede dar un desarrollo más completo de las funciones cognitivas que demanda una determinada profesión. No obstante, dicha teoría enfrenta retos para poder explicar cómo se podría correlacionar un EC profesional amplio y versátil con un set de funciones específicas localizadas topográficamente.

Finalmente, las correlaciones observadas entre la edad y el apartado de dudas/ inconsistencias del Explora mostraron dos tendencias importantes: una que sugiere que, a menor edad, más dudas en las respuestas de EC profesional, y otra en la que, a mayor edad, más alto es el grado de inconsistencias en las respuestas. Probablemente estos resultados remiten a que los adolescentes tienen menos claro qué quieren estudiar en edades tempranas y a su vez, la carga de responsabilidad que conlleva esta decisión es más alta en los adolescentes de mayor edad, quienes empiezan a considerar diversas opciones de profesionalización sin mucha claridad sobre los pro y contra de cada carrera. Al respecto, vale la pena mencionar que las investigaciones muestran que los adolescentes, en general, tienden a experimentar cargas de duda y ansiedad con respecto a la elección profesional (Bravo y Vergara 2018).

\section{Conclusiones}

Los resultados de este trabajo sugieren una relación entre los EC profesionales y perfiles de dominancia cerebral. Estos resultados muestran una tendencia consistente con la teoría y estudios previos con respecto a la especialización funcional del cerebro. En este sentido, es necesario ampliar el espectro de los datos para el análisis e incluir medidas que involucren tecnología de registro biológico como RMF o electroencefalografía, y de esta forma, realizar una correlación funcional que puede ir más allá de lo teórico.

De igual manera, se hace necesario ampliar el tamaño de la muestra para considerar la 
generalización de los resultados e incluir análisis con otras variables sociodemográficas como nivel de ingreso, con el fin de determinar otros aspectos que podrían tener incidencia en la elección profesional.

La elección profesional es un área de estudio que tiene potencial para la aplicación de los más recientes avances teóricos y metodológicos de la neurociencia y puede tener un alto impacto en la manera en que se concibe dicha decisión, el logro académico y profesional.

\section{Referencias}

Aberg, K. C., Doell, K. C., y Schwartz, S. (2016). The "Creative Right Brain" Revisited: Individual Creativity and Associative Priming in the Right Hemisphere Related to Hemispheric Asymmetries in Reward Brain Function. Cerebral Cortex, 27(10), 4946-4959. https://doi.org/10.1093/ cercor/bhw288

Bendall, R. C., Galpin, A., Marrow, L. P., y Cassidy, S. (2016). Cognitive Style: Time to Experiment. Frontiers in Psychology, 7. https://doi. org/10.3389/fpsyg.2016.01786

Blazhenkova, O., y Kozhevnikov, M. (2009). The new object-spatial-verbal cognitive style model: Theory and measurement. Applied Cognitive Psychology, 23, 638-663.

Bravo, G., y Vergara, M. (2018). Factores que determinan la elección de carrera profesional en estudiantes de undécimo grado de colegios públicos y privados de Barrancabermeja. Revista Psicoespacios, 12(20), 35-48 https://doi. org/10.25057/issn.2145-2776

Coffield, F., Moseley, D., Hall, E., y Ecclestone, K. (2004). Learning Styles and Pedagogy in Post16 Learning. Learning Skills Research Centre.

Congreso de la República (2006). Ley 1090 de 2006, por la cual se reglamenta el ejercicio de la profesión de Psicología, se dicta el Código Deontológico y Bioético y otras disposiciones. Recuperado de http://www.colpsic. org.co/quienes-somos/ ley-1090-de-2006/182
Cools, E. (2009). A reflection on the future of the cognitive style field: a proposed research agenda. Reflect. Educ. 5, 19-34.

Ettinger-Veenstra, H. V., Ragnehed, M., Hällgren, M., Karlsson, T., Landtblom, A., Lundberg, P., y Engström, M. (2010). Right-hemispheric brain activation correlates to language performance. Neurolmage, 49(4), 3481-3488. https://doi. org/10.1016/j.neuroimage.2009.10.041

Figueroa Pilz, A., y Ortega Olivares, M. (2010). Condición de género y elección profesional. El área de físico-matemático en las mujeres. Investigación y Ciencia, 18(46),18-27. https://www.redalyc.org/articulo.oa?id=67413508004

Fountoulakis, K. N., Nimatoudis, I., y Gonda, X. (2019). The Human Connectome: Functional Anatomy of the Brain. Psychobiology of Behaviour, 1-48. https://doi.org/10.1007/978-3-030-18323-3_1

Gardié, O. (2000). Determinación del perfil de estilos de pensamiento y análisis de sus posibles implicaciones en el desempeño de profesionales universitarios venezolanos. Estudios Pedagógicos, (26), 25-38.

Genovese (2005) Hemispheric Cognitive Style: A Comparison of Three Instruments, The Journal of Genetic Psychology: Research and Theory on Human Development, 166(4), 467-481, https://doi. org/10.3200/GNTP.166.4.467-482

Goldberg, E. (2019). Creatividad: el cerebro humano en la era de la innovación. Planeta, Drakontos.

Gottfredson, L. S. (2003). The Challenge and Promise of Cognitive Career Assessment. Journal of Career Assessment, 11(2), 115-35. https://doi. org/10.1177/1069072703011002001

Herrmann, N. (1991). The Creative Brain. Training and Development Journal, 25(4), 275-295. https:// doi.org/10.1002/j.2162-6057.1991.tbo1140.x

Herrmann, N. (2009). Measurement of brain dominance. Hermann International Solutions. http://resources.herrmannsolutions.com.au/ resources/ArticleMeasurementBrain Dominance.pdf 
Höffler, T. N., KO Januchta, M., y Leutner, D. (2017). More Evidence for Three Types of Cognitive Style: Validating the ObjecT Spatial Imagery and Verbal Questionnaire Using Eye Tracking when Learning with Texts and Pictures. Appl. Cognit. Psychol.,31:109-115. https://doi.org/10.1002/ acp. 3300

Holland, J. L. (1992). Making vocational choices: A theory of vocational personalities and work environments ( $2^{\underline{a}}$ ed.). Psychological Assessment Resources.

Holland, J. L. (1997). Making vocational choices: A theory of vocational personalities and work environments ( $3^{\underline{a}}$ ed.). Psychological Assessment Resources.

Jiménez, C. A. (2000). Cerebro creativo y lúdico. Hacia la construcción de una nueva didáctica para el siglo XXI. Cooperativa Editorial Magisterio.

Kanske, P. (2018). The social mind: Disentangling affective and cognitive routes to understanding others. Interdisciplinary Science Reviews, 43(2), 115-124. https://doi.org/10.1080/03080188.201 8.1453243

Khalil, R., Godde, B., y Karim, A. A. (2019). The Link Between Creativity, Cognition, and Creative Drives and Underlying Neural Mechanisms. Frontiers in Neural Circuits, 13. https://doi.org/10.3389/ fncir.2019.00018

Kozhevnikov, M. (2007). Cognitive styles in the context of modern psychology: Toward an integrated framework of cognitive style. Psychological Bulletin, 133(3), 464-481. https://doi. org/10.1037/0033-2909.133.3.464

Ko-Januchta, M., Höffler, T., Thoma, G.-B., Prechtl, H., y Leutner, D. (2017). Visualizers versus verbalizers: Effects of cognitive style on learning with texts and pictures - An eye-tracking study. Computers in Human Behavior, 68, 170-179. https:// doi.org/10.1016/j.chb.2016.11.028

Kraemer, D. J. M., Rosenberg, L. M., y Thompson-Schill, S. L. (2009). The neural correlates of visual and verbal cognitive styles. J. Neurosci. 29, 3792-3798. https://doi.org/10.1523/JNEUROS$\mathrm{Cl} .4635-08.2009$
Kroger, J. K., Nystrom, L. E., Cohen, J. D., y Johnson-Laird, P. N. (2008). Distinct neural substrates for deductive and mathematical processing. Brain Research, 1243, 86-103. https://doi.org/10.1016/j. brainres.2008.07.128

Martínez-Vicente, J. M. (2016). Explora cuestionario para la orientación vocacional y profesional. International Journal of Developmental and Educational Psychology. Revista INFAD De Psicología, 4(1), 335. https://doi.org/10.1706o/ ijodaep.2014.n1.v4.620

Martínez-Vicente, J. M. (2019). La orientación y el asesoramiento vocacional en educación secundaria a través del explora (cuestionario para la orientación vocacional y profesional). En Salud y ciclo vital (pp. 71-78). Dykinson. https:// doi.org/10.2307/j.ctvfb6z2h.10

Martínez-Vicente, J.M., y Santamaría, P. (2013). Explora. Cuestionario para la orientación vocacional y profesional. TEA Ediciones.

Navarro Guzmán C., y Casero Martínez A. (2012). Análisis de las diferencias de género en la elección de estudios universitarios. Estudios sobre educación, 22, 115-132. hdl.handle. net/10171/22628

Quinn, J. (2013). Drop-out and completion in higher education in Europe. Neset. https://doi. org/10.2766/085754

Ramiro, P., Navarro, J. I., Menacho, I., y Aguilar, M. (2010). Cognitive style. Revista Latinoamericana de Psicología, 42(2), 193-202. http:// www.scielo.org.co/scielo.php?script $=$ sci_arttext \& pid =S0120-05342010000200003\&l$\mathrm{ng}=\mathrm{en} \& \mathrm{tlng}=\mathrm{en}$

Rodríguez Méndez, M. C., Peña Calvo, J., y Pérez García O. (2016). Estudio cualitativo de las diferencias de género en la elección de opciones académicas en los estudiantes del bachillerato científico-técnico. Teoría de la educación, 28, (1), 189-207. http://dx.doi.org/10.14201/teoredu2016281189207

Rogers, L. (2001). Sexing the brain. Columbia University Press. 
Schurz, M., Radua, J., Aichhorn, M., Richlan, F., y Perner, J. (2014). Fractionating theory of mind: A meta-analysis of functional brain imaging studies. Neuroscience \& Biobehavioral Reviews, 42, 9-34. http://dx.doi.org/10.1016/j.neubiorev.2014.01.009

Schurz, M., Radua, J., Aichhorn, M., Richlan, F., y Perner, J. (2014). Fractionating theory of mind: a meta-analysis of functional brain imaging studies. Neurosci. Biobehav. Rev., 42, 9-34. https://doi. org/10.1016/j.neubiorev.2014.01.009

Shi, B., Cao, X., Chen, Q., Zhuang, K., y Qiu, J. (2017). Different brain structures associated with artistic and scientific creativity: A voxel-based morphometry study. Scientific Reports, 7(1). http:// dx.doi.org/10.1038/srep42911

Shin, G., y Kim,C. (2015). Neural correlates of cognitive style and flexible cognitive control. Neuroimage 113,78-85. http://dx.doi.org/10.1016/j.neuroimage.2015.03.046

Spadies (2019). Sistema para la Prevención de la Deserción en las Instituciones de Educación Superior. https://www.mineducacion.gov.co/sistemasdeinformacion/1735/w3-article-363411.html

Stietz, J., Jauk, E., Krach, S., y Kanske, P. (2019). Dissociating Empathy From Perspective-Taking: Evidence From Intra- and Inter-Individual Differences Research. Frontiers in Psychiatry, 10. http:// dx.doi.org/10.3389/fpsyt.2019.00126
Tinajero Vacas, C., y Páramo Fernández, M. F. (2013). El estilo cognitivo dependencia-independencia en el proceso de enseñanzaaprendizaje. Revista Colombiana de Educación, (64), 57-78. http://www.scielo.org.co/ scielo.php?script $=$ sci_arttext $\&$ pid $=$ So120$39162013000100003 \& \operatorname{lng}=$ en\&tlng $=$.

Velásquez Burgos, B. M., Remolina de Cleves, N., y Calle Márquez, M. G. (2013). Análisis correlacional del perfil de dominancia cerebral de estudiantes de ciencias de la salud y estudiantes de ciencias sociales de la Universidad Colegio Mayor de Cundinamarca. Nova, 11(20), 71-82. http://www.scielo.org.co/ scielo.php?script $=$ sci_arttext $\&$ pid $=\mathrm{S} 1794^{-}$ $24702013000200008 \& \operatorname{lng}=$ en\&tlng $=$.

Yarlequé, Ch., Navarro, L. G., Núñez, E. L., Padilla, M. S., y Álvarez G.S. (2018) Perfil de dominancia cerebral en ingresantes a la universidad de Huancayo Horizonte de la Ciencia, 8(15) http://revistas. uncp.edu.pe/index.php/horizontedelaciencia/ article/view/269

Zumárraga-Espinosa, M., Castro, M., Escobar, P., Boada, M., Peña Herrera, L., González, Y., Romero, J., Luzuriaga, J., y Armas, R. (2018). Afinidad entre intereses profesionales y carrera elegida: Un análisis de su relación con la deserción universitaria temprana [ponencia]. Congresos CLABES VIII. Ciudad de Panamá https://revistas.utp. ac.pa/index.php/clabes/article/view/1951 